\title{
A Computational Method For Fuzzy ARITHMETIC OPERATIONS
}

\author{
Thowhida Akther ${ }^{1}$ and Sanwar Uddin Ahmad ${ }^{2}$ \\ ${ }^{1}$ Faculty of Science and Information Technology \\ Daffodil International University, Dhaka, Bangladesh \\ ${ }^{2}$ Department of Mathematics \\ University of Dhaka, Dhaka, Bangladesh \\ E-mail: lizudu@yahoo.com and sanwardu@gmail.com
}

\begin{abstract}
In this paper, a computer implementation to evaluate the arithmetic operations on two fuzzy numbers with linear membership functions has been developed. The fuzzy arithmetic approached by the interval arithmetic is used here. The algorithm of the developed method with a numerical example is also provided. Using this method four basic arithmetic operations between any two TFNs can be evaluated without complexity.
\end{abstract}

Keywords: Fuzzy arithmetic, Fuzzy number, Membership Function, Interval arithmetic, $\alpha$ cut.

\section{Introduction}

The fuzzy numbers were introduced by [1] and frequently arise in decision making, control theory, fuzzy systems and approximate reasoning problems. The fuzzy arithmetic was developed by several later authors, e.g. [2 - 4].

In recent years some research on the application of the fuzzy set theory [5] in education, based on the concepts of fuzzy numbers and fuzzy arithmetic has begun e.g., $[6,7]$. Fuzzy numbers are also used in statistics, computer programming, engineering (especially communications), and experimental science.

In calculations generally for computational efficiency many applications limit the membership functions of the fuzzy numbers to triangular or trapezoidal fuzzy numbers. Using the inverse function [4] the implementation of arithmetic operations on fuzzy numbers is computationally complex and the implementation of the extension principle [5] is equivalent to solving a nonlinear programming problem. A direct, fast and accurate way of computing the arithmetic operations on fuzzy numbers has engaged researchers and students of many fields and became the motivation to produce the proposed computational method. The users can evaluate the exact form of the membership functions of the resultant fuzzy numbers in a simple and accurate implementation on computer using MATHEMATICA.

In this paper the triangular fuzzy numbers (TFN) are considered. The fuzzy arithmetic approached by interval arithmetic for fuzzy numbers has been used in this paper. In recent times [8] proposed a method using spreadsheet to evaluate the arithmetic operations on fuzzy numbers and [9] proposed a method using MATHEMATICA to evaluate the multiplication on fuzzy numbers. In this paper the method proposed in [9] is extended for all the four basic arithmetic operations on fuzzy numbers and evaluate the exact forms of the membership functions of the fuzzy results.

\section{Fuzzy Intervals and Numbers}

The universe of discourse on which fuzzy numbers are defined is the set of real numbers and its subsets (e.g., integers or natural numbers), its membership function should assign the degree of 1 to the central value and degrees to other numbers that reflects their proximity to the central value according to some rule therefore the membership functions ought to be normal and convex. The membership should thus decrease from 1 to 0 on both sides of the central value. Fuzzy sets of this kind are known as fuzzy numbers. Membership functions that conform to this intuitive conception must be expressed in the general form [10], 


$$
A(x)= \begin{cases}f(x), & \text { for } x \in[a, b] \\ 1, & \text { for } x \in[b, c] \\ g(x), & \text { for } x \in[c, d] \\ 0, & \text { otherwise }\end{cases}
$$

where $a \leq b \leq c \leq d$, $f$ is increasing and rightcontinuous function on $[a, b]$, and $g$ is decreasing and left-continuous function on $[c$, d]. If $b=c$ then $A$ is a fuzzy number otherwise it is known as fuzzy interval. When $f(x)=\frac{x-a}{b-a}$ and $g(x)=\frac{d-x}{d-c}$ the fuzzy number $A$ is a trapezoidal fuzzy number. When $f$ and $g$ are as before and $b=c$ then $A$ is a triangular fuzzy number (TFN).

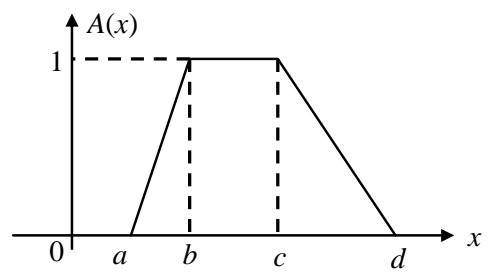

Fig. 1 A trapezoidal fuzzy number

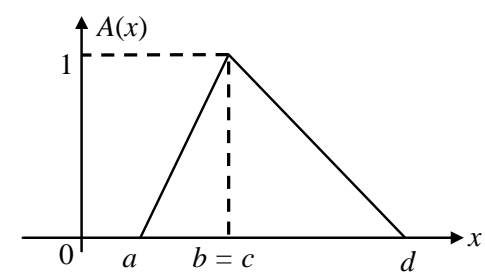

Fig. 2 A triangular fuzzy number

The TFN $A$ is denoted by $A=<a, b, d>$. The $\alpha$-cuts of the TFN $A$ are

$[a+\alpha(b-a), d-\alpha(d-b)], \forall \alpha \in[0,1]$

\section{Standard Fuzzy Arithmetic Operations}

The essential properties for defining meaningful arithmetic operations on fuzzy numbers are,

(a) Fuzzy numbers are normal fuzzy sets, i.e. $\exists x \in X$ s.t. $A(x)=1$.

(b) The $\alpha$-cuts of every fuzzy number are closed intervals of real numbers $\forall \alpha \in(0,1]$.

(c) The support of every fuzzy number, $S(A)$ $=\operatorname{Supp}(\mathrm{A})=\{x \in \mathrm{IR} \mid A(x)>0\}$ is bounded.

(d) Fuzzy numbers are convex fuzzy sets i.e. for all $x_{1}, x_{2} \in X$,

$A\left(\lambda x_{1}+(1-\lambda) x_{2}\right) \geq \min \left(A\left(x_{1}\right), A\left(x_{2}\right)\right)$.

For computing the four basic arithmetic operations on fuzzy numbers we represent the numbers by their $\alpha$-cuts and employ interval arithmetic to the $\alpha$-cuts. Consider two arbitrary fuzzy numbers $\mathrm{A}$ and $\mathrm{B}$, and let $\otimes$ denote any of the four arithmetic operations. Then, for each $\alpha \in(0,1]$, the $\alpha$-cut of $A \otimes B$ is defined in terms of the $\alpha$-cuts of $A$ and $B$ by the formula

$$
{ }^{\alpha}(A \otimes B)={ }^{\alpha} A \otimes{ }^{\alpha} B
$$

which is not applicable when $\otimes$ is division and $0 \in^{\alpha} B$ for any $\alpha \in(0,1]$. Once the $\alpha$-cuts ${ }^{\alpha}(A \otimes B)$ are determined, the resulting fuzzy number $A \otimes B$ is readily expressed by the decomposition theorem of fuzzy sets as,

$$
A \otimes B=\bigcup_{\alpha \in[0,1]}^{\alpha}(A \otimes B) \cdot \alpha
$$

Since every fuzzy set is uniquely represented by its $\alpha$-cuts and these are closed intervals of real numbers, arithmetic operations on fuzzy numbers can defined in terms of arithmetic operation on closed intervals of real numbers which are described in the next section.

\section{Interval Arithmetic}

The arithmetic operations for intervals [11, 12], based on the respective real arithmetic operations at the extremes of the intervals are defined as follows,

Let $T=[a, b]$ and $S=[c, d]$ be two closed intervals of real numbers, then

$\mathrm{T} \otimes \mathrm{S}=\{x$ : there is some $y$ in $T$, and some $z$ in $S$, such that $x=y \otimes z$, where $\otimes$ is the arithmetic operations except division (/), which is not defined if $0 \in S$. The arithmetic operations on intervals are defined in the ends of their intervals, as is shown below [11],

Addition (+):

$T+S=[a, b]+[c, d]=[a+c, b+d]$

Subtraction (-):

$T-S=[a, b]-[c, d]=[a-d, b-c]$

\section{Multiplication (.):}

$T . S=[a, b] .[c, d]=[\min \{a c, a d, b c, b d\}$, $\max \{a c, a d, b c, b d\}]$

More precisely the extremes of the multiplied interval is provided in the following table 1.

Division (/):

$[a, b] /[c, d]=\left[\min \left(\frac{a}{c}, \frac{a}{d}, \frac{b}{c}, \frac{b}{d}\right)\right.$,

$\left.\max \left(\frac{a}{c}, \frac{a}{d}, \frac{b}{c}, \frac{b}{d}\right)\right]$, provided $0 \notin[c, d]$.

The extremes of the resultant interval are given in the following table 2 . 
Table 1 Table for computing the extremes of the product of two closed intervals

\begin{tabular}{|c|c|c|}
\hline $\mathrm{T}=[a, b]$ & $\mathrm{S}=[c, d]$ & $\mathrm{T} \cdot \mathrm{S}$ \\
\hline \multirow{3}{*}{$0 \leq a \leq b$} & $0 \leq c \leq d$ & {$[a c, b d]$} \\
\cline { 2 - 3 } & $c \leq 0 \leq d$ & {$[b c, b d]$} \\
\cline { 2 - 3 } & $c \leq d \leq 0$ & {$[b c, a d]$} \\
\hline \multirow{3}{*}{$a \leq 0 \leq b$} & $0 \leq c \leq d$ & {$[a d, c d]$} \\
\cline { 2 - 3 } & $c \leq 0 \leq d$ & {$[\min (a d, b c)$,} \\
& & $\max (a c, b d)]$ \\
\cline { 2 - 3 }$a \leq b \leq 0$ & $c \leq d \leq 0$ & {$[b c, a c]$} \\
\hline \multirow{3}{*}{} & $0 \leq c \leq d$ & {$[a d, b c]$} \\
\cline { 2 - 3 } & $c \leq 0 \leq d$ & {$[a d, a c]$} \\
\cline { 2 - 3 } & $c \leq d \leq 0$ & {$[b d, a c]$} \\
\hline
\end{tabular}

Table 2 Table for computing the extremes of the division of two closed intervals

\begin{tabular}{|c|c|c|}
\hline$T=[a, b]$ & $S=[c, d]$ & $T / S$ \\
\hline $0 \leq a \leq b$ & $0<c \leq d$ & {$\left[\frac{a}{d}, \frac{b}{c}\right]$} \\
\hline \multirow{2}{*}{$a \leq 0 \leq b$} & $c \leq d<0$ & {$\left[\frac{b}{d}, \frac{a}{c}\right]$} \\
\hline \multirow{2}{*}{$a \leq b \leq 0$} & $c \leq d<0$ & {$\left[\frac{a}{c}, \frac{b}{c}\right]$} \\
\cline { 2 - 3 }$a \leq c \leq d$ & {$\left[\frac{b}{d}\right]$} \\
\hline \multirow{2}{*}{$a \leq c \leq d$} & {$\left[\frac{a}{c}, \frac{b}{d}\right]$} \\
\hline & $c \leq d<0$ & {$\left[\frac{b}{c}, \frac{b}{d}\right]$} \\
\hline
\end{tabular}

\section{Algorithm for the proposed method}

In this section the algorithm of the developed MATHEMATICA code for the fuzzy arithmetic of two TFNs which evaluate the membership function of the resultant fuzzy number is provided.

Given $A=<a_{1}, f_{\mathrm{A}}, b_{1}, g_{\mathrm{A}}, c_{1}>, B=<a_{2}, f_{\mathrm{B}}, b_{2}$, $g_{\mathrm{B}}, c_{2}>$.

Input:

1. The real numbers $a_{1}, b_{1}, c_{1}, a_{2}, b_{2}, c_{2}$.

2. The functions $f_{\mathrm{A}}(x), x \in\left[a_{1}, b_{1}\right], g_{\mathrm{A}}(x)$, $x \in\left[b_{1}, c_{1}\right], f_{\mathrm{B}}(x), x \in\left[a_{2}, b_{2}\right], g_{\mathrm{B}}(x)$, $x \in\left[b_{2}, c_{2}\right]$.

Output: Evaluate the analytical form of the membership function of $(A+B)(x),(A-B)(x)$, $(A . B)(x),(A / B)(x)$.

Step 1. Find ${ }^{\alpha} A=\left[{ }^{\alpha} A_{1},{ }^{\alpha} A_{2}\right]$,

${ }^{\alpha} B=\left[{ }^{\alpha} B_{1},{ }^{\alpha} B_{2}\right]$ in terms of $\alpha$.

If $\otimes=$ Addition,

Then ${ }^{\alpha}(A+B)=\left[{ }^{\alpha} A_{1}+{ }^{\alpha} B_{1},{ }^{\alpha} A_{2}+{ }^{\alpha} B_{2}\right]$ by Section 4 and go to Step2.
If $\otimes=$ Subtraction, Then

${ }^{\alpha}(A-B)=\left[{ }^{\alpha} A_{1}-{ }^{\alpha} B_{2},{ }^{\alpha} A_{2}-{ }^{\alpha} B_{1}\right]$ by Section 4 and go to Step2.

Step 2. Using backward transformation technique, compute the membership functions of $(A \otimes B)(x)$ in terms of $x$ for $x \in\left[{ }^{\alpha}(A \otimes B)_{1}(0),{ }^{\alpha}(A \otimes B)_{1}(1)\right] \quad$ and $x \in\left[{ }^{\alpha}(A \otimes B)_{2}(0),{ }^{\alpha}(A \otimes B)_{2}(1)\right]$ respectively.

If $\otimes=$ Division and $0 \notin\left[{ }^{\alpha} B_{1},{ }^{\alpha} B_{2}\right]$ Then go to Step3

Else Output "Division is not defined"

End If.

If $\otimes=$ Multiplication [9] Then go to Step3

Step 3. Define functions to compute the extremes ${ }^{\alpha}(A \otimes B)_{1},{ }^{\alpha}(A \otimes B)_{2}$ of ${ }^{\alpha}(A \otimes B)$, for $\alpha \in[m, n]$ in terms of $\alpha, m, n$.

Step 4. Define functions using backward transformation technique, which compute the membership functions of $(A \otimes B)(x)$ in terms of $x$ for $x \in\left[{ }^{\alpha}(A \otimes B)_{1}(m),{ }^{\alpha}(A \otimes B)_{1}(n)\right]$ and $x \in\left[{ }^{\alpha}(A \otimes B)_{2}(m),{ }^{\alpha}(A \otimes B)_{2}(n)\right]$ respectively.

Step 5. Let $K_{1}=\operatorname{Min}\{A(0), B(0)\}$ and $K_{2}=\operatorname{Max}\{A(0), B(0)\}$.

If $\otimes=$ Division go to Step 5 .

If $\otimes=$ Multiplication [9] go to Step 6 .

\section{Step 6.}

Case1. If $K_{1}=0$ or $K_{2}=1$

Compute $(A / B)(x)$ for $0<\alpha<1$.

Case2. If $0<K_{1}<1$

Compute $(A / B)(x)$ for $0<\alpha<K_{1} \quad$ and $K_{1}<\alpha<1$ respectively.

Step 7.

Case1. If $K_{1}=0$ and $0<K_{2}<1$

Compute $(A . B)(x)$ for $0<\alpha<K_{2}$, and $K_{2}<\alpha<1$ respectively.

Case2. If $\left(K_{1}=0\right.$ and $\left.K_{2}=1\right)$ or $K_{2}=0$

Compute $(A . B)(x)$ for $0<\alpha<1$.

Case3. If $K_{1}>0$ and $0<K_{2}<1$

Compute $(A . B)(x)$ for $0<\alpha<K_{1}$, $K_{1}<\alpha<K_{2}$ and $K_{2}<\alpha<1$ respectively.

Case4. If $K_{1}=1$. 
Compute (A.B) $(x)$ for $0<\alpha<1$.

Case5. If $0<K_{1}<1$ and $K_{2}=1$

Compute $(A . B)(x)$ by Case3

\section{Numerical Example}

Consider two TFN's with membership functions

$$
\begin{aligned}
& A(x)= \begin{cases}\frac{x+1}{2}, & -1 \leq x \leq 1 \\
\frac{3-x}{2}, & 1 \leq x \leq 3, \\
0, & \text { otherwise }\end{cases} \\
& \text { and } B(x)= \begin{cases}\frac{x-1}{2}, & 1 \leq x \leq 3 \\
\frac{5-x}{2}, & 3 \leq x \leq 5 \\
0, & \text { otherwise }\end{cases}
\end{aligned}
$$

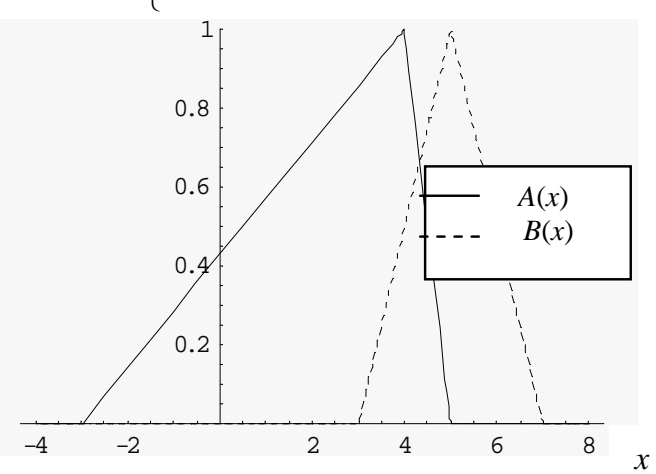

Fig. 3 Membership functions of $A(x)$ and $B(x)$

The membership function for the addition of the two TFN's is $\operatorname{add} A B(x)= \begin{cases}\frac{12-x}{3}, & 9<x \leq 12 \\ \frac{x}{9}, & 0 \leq x \leq 9\end{cases}$

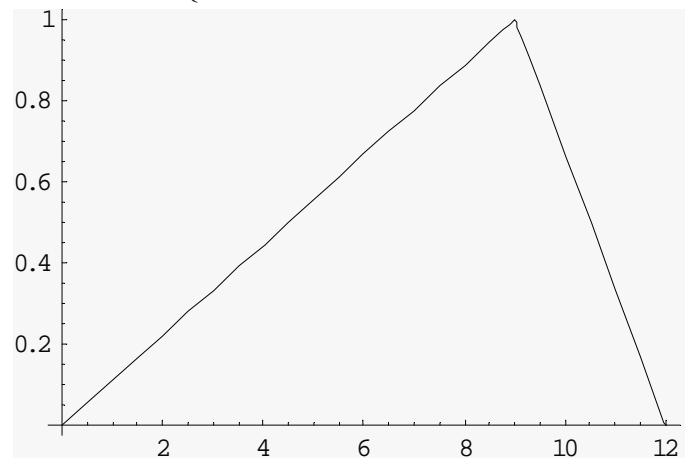

Fig. 4 Membership function of $(A+B)(x)$
The membership function for the subtraction of the two TFN's is $\operatorname{subAB}(x)= \begin{cases}\frac{2-x}{3}, & -1<x \leq 2 \\ \frac{10+x}{9}, & -10 \leq x \leq-1\end{cases}$

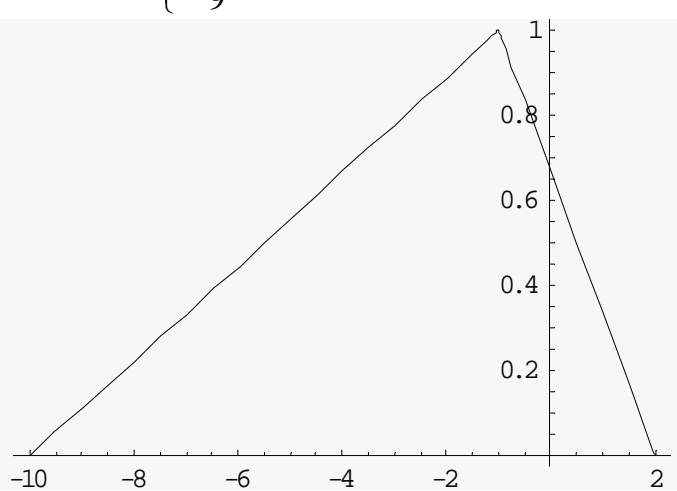

Fig. 5 Membership function of $(A-B)(x)$

The membership function for the product of the two TFN's is

$$
A B(x)= \begin{cases}\frac{1}{28}(55-\sqrt{1849-56 x}), & -21 \leq x \leq 0 \\ \frac{1}{4}(17-\sqrt{9+8 x}), & 20<x \leq 35 \\ \frac{1}{28}(-15+\sqrt{729+56 x}), & 0<x \leq 20\end{cases}
$$

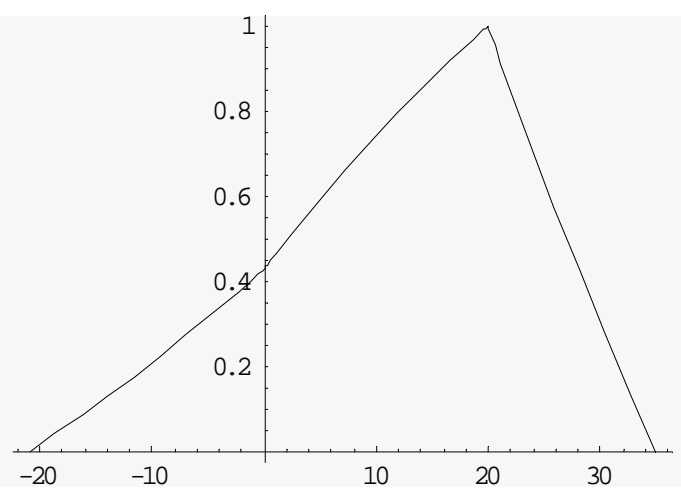

Fig. 6 Membership function of $(A . B)(x)$

The membership function for the division of the

$$
\operatorname{div} A B(x)= \begin{cases}-\frac{3(1+x)}{-7+2 x}, & -1 \leq x \leq 0 \\ \frac{5-3 x}{1+2 x}, & \frac{4}{5}<x \leq \frac{5}{3} \\ \frac{3+7 x}{7+2 x}, & 0<x \leq \frac{4}{5}\end{cases}
$$




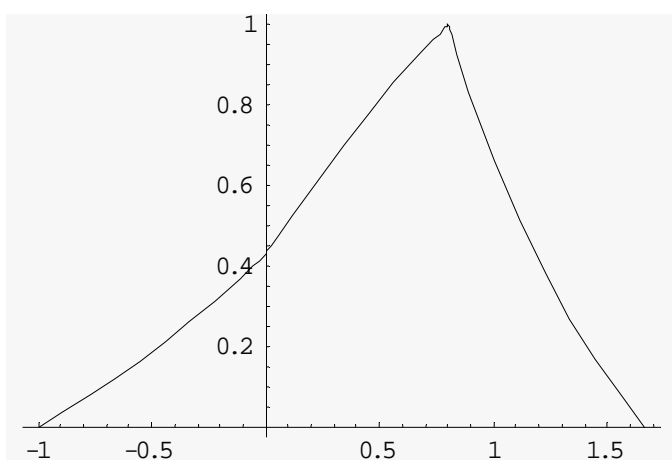

Fig. 7 Membership function of $(A / B)(x)$

\section{Conclusions}

This paper presents a direct, fast and accurate way of computing the arithmetic operations on fuzzy numbers as well as the analytic form of the resultant membership functions. The implementation proposed by [8] is limited in finding the membership grade of a particular number, provides no information about the resultant fuzzy number (e.g. the shape, the support etc.), while in [9] the authors implemented the method only for the multiplication of two TFN's with linear membership function. This paper is the extension of [9], where the implementation for addition, subtraction and division of two TFN's is provided. Users can evaluate the four basic arithmetic operations between any two TFNs without complexity and even plot the membership function of the resultant fuzzy number to take any further decisions (e.g., shape, support, $\alpha$-cuts etc.).

\section{References}

[1] L. A. Zadeh, "The Concept of linguistic variable and its applications to approximate reasoning", Inform. Sci. Vol. 8, 1975, pp. 199-251.

[2] M. MizuMoto, K. Tanaka, "The four operations on fuzzy numbers", Syst. Compute. Controls, Vol. 7, 1976, pp. 73-81.

[3] D. Dubois, H. Prade, "Operation on fuzzy numbers”, Int. J. Syst. Sci., Vol. 9, 1978, pp. 613626.

[4] R. R. Yager, "On the lack of inverses in fuzzy arithmetic”, Fuzzy Sets Syst., Vol. 4, 1980, pp. 7382.
[5] L.A. Zadeh, "Fuzzy Sets", Information and Control, Vol. 8, 1965, pp. 338-353.

[6] Chih-Huang Wang, Shy-Ming Chen, "Appraising the performance of high school teachers based on fuzzy number arithmetic operations", Soft Comput., Vol. 12, 2008, pp. 919-934.

[7] Law CK, "Using fuzzy numbers in education grading system”, Fuzzy Sets Syst., Vol. 83, No. 3, 1996, pp. 311-323.

[8] P.S. Kechagias, Basil K. Papadopoulos, "Computational method to evaluate fuzzy arithmetic operations”, Applied Mathematics and Computations, Vol. 185, 2007, pp. 169-177.

[9] Sanwar Uddin Ahmad and Thowhida Akther, "Multiplication of Fuzzy Numbers Using Mathematica”, Daffodil Int. Univ. J. of Sc. \& Tech., Vol. 3, Issue 1, pp 32 - 35, 2008.

[10] G.J.Klir, B.Yuan, Fuzzy Sets and Fuzzy Logic: Theory and Applications, Prentice-Hall PTR, Upper Saddlie River, 1995.

[11] Moore, R. E., Interval Analysis, Prentice Hall, New Jersey, 1966.

[12] Oliveira, P. W. de., "Fundamentos de Matemática Intervalar", $1^{\text {a }}$ ed. Instituto de Informática da UFRGS: SAGRA-Luzzato, 1997.

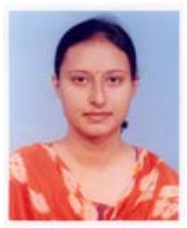

Thowhida Akther has received her MS in Applied Mathematics and B.Sc (hons) in Mathematics from University of Dhaka. Now she is working as a Lecturer under the Department of CSE, CIS \& CS in Daffodil International University, Dhaka.

Her interested field of research includes Numerical Methods, Operations Research and Fuzzy Mathematics.

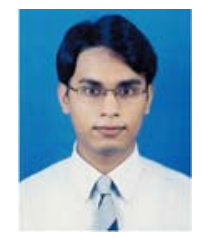

Sanwar Uddin Ahmad has completed MS in Applied Mathematics and B.Sc (hons) in Mathematics from University of Dhaka. Now he is working as a Lecturer in the Department of Mathematics, University of Dhaka.

His areas of research are Operations Research, Fuzzy Mathematics and Group Theory. 